\title{
THE NATIONAL PHYSICAL LABORATORY
}

$\mathrm{T}$ HE National Physical Laboratory held two open days, on May 14 and 15 . One hundred and eighty exhibits were on view to 3,250 guests of the Laboratory, who came from industry, the universities and Government departments. It was the first time that the Laboratory had been on show since the divisional reorganization, announced last February. This year, as an experiment, the range of exhibits was somewhat restricted, because the items chosen were selected to display work in certain chosen topics. For example, the exhibits shown by the Light Division all fell into three topics : optical instruments and methods; standards and measurements of radiation, light and colour ; and research on vision. Work which did not fit into the topics selected was, therefore, not on display, but arrangements were made so that it could be discussed with the responsible officers of the Laboratory.

The Aerodynamies Division is investigating the problems of hypersonic flow, that is, at speeds in excess of Mach 5. Several new aerodynamic problems arise at such speeds, including intense aerodynamic heating resulting in dissociation of the working gases and, if the temperature is high enough, their ionization. For this investigation, shock-tubes are being developed which operate for about half a millisecond; these obviate the problems of overheating the equipment. Surface temperatures and pressures are measured with rapid-response instruments and a schlieren optical system has been developed which records flow-density patterns around the test bodies using a camera of the Cranz-Schardin type (see Fig. 1). In addition to the shock-tube being used for the main programme of research, two small tubes have been constructed for work of a more fundamental nature on the properties of gases at high temperatures. One of these will be used to study the visible radiation spectroscopically in order to evaluate the gas temperature, the other to investigate the electron concentrations by means of micro-wave techniques. Some of the non-aeronautical work of the Division was displayed in the form of a small exhibition of models which had been tested in low-speed wind tunnels. There is a considerable demand for accurate information on the effects of wind on structures such as the B.B.C. television transmitting tower, suspension bridges, chimney stacks, blocks of houses and the aerial system of the University of Manchester radio telescope at Jodrell Bank. Similar work is carried out on the airflow around ships and buildings to ensure that the effluent from funnels and chimneys causes neither annoyance nor damage.

The Control Mechanisms and Electronics Division displayed a conditional probability computer. This is a form of 'learning machine'. It has five input channels and thirty-one units to store statistical information of the patterns presented by the inputs. When some statistical data have been accumulated in the storage units and one or more of the input channels is activated, the computer determines from the data whether such activation is usually accompanied by activity in one or more of the other input channels. If it is, the computer 'infers' that this activity is again present. The units which store the statistical information are counters which function by charging capacitors. These capacitors have deliberate slow leaks in parallel with them so that the computer's inferences are based more on later events than earlier ones. The demonstration showed the computer controlling a small mobile carriage which followed the boundary between black and white areas painted on the surface on which the carriage ran. The black and white areas could be effectively interchanged by reversing the steering mechanism, so making the computer learn the new conditions. It is hoped to develop the computer to a stage when it can function as an industrial control mechanism which will select optimum conditions by a trial-and-error learning process.

The Light Division has made significant advances in the techniques necessary to produce cheap, metrologically accurate diffraction gratings used for automatic machine-tool control. Gratings several feet long are being made by a photographic printing method of reproduction using the fine-grain emulsions already available in the Laboratory. Photographic techniques also open up the possibility of producing gratings directly from an interference pattern, so removing many of the possible sources of error in manufacture. The regularity of spacing would then depend entirely on the accuracy of the interferometer mirrors and the stability of the photographic emulsion during processing. This quite old idea, due originally to Michelson, has been made practicable by the development of both fine-grain emulsions and isotope discharge tubes used as monochromatic sources.

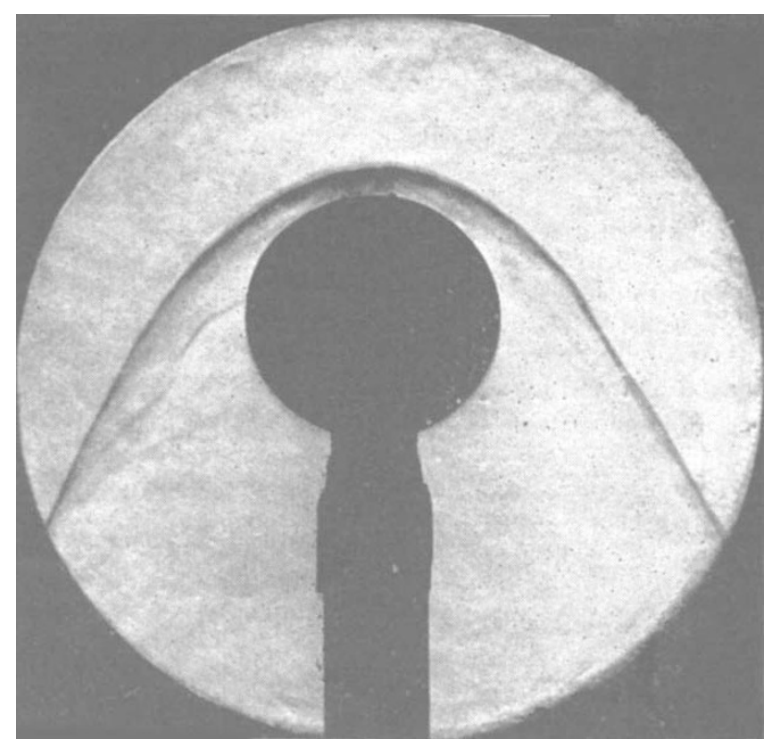

Fig. 1. Shock wave of flow around a sphere in the National Physical Laboratory hypersonic shock tube. Conditions (approximate): Mach 9 for equivalent altitude of $150,000 \mathrm{ft}$. and stagnation temperature of $6,000^{\circ} \mathrm{K}$. (Crown copyright reserved) 


\section{№. 4627 July 5,1958}

Gratings 3 in. square with 30,000 lines per inch have been produced. Two gratings made in this, or a more conventional, way can then be arranged to produce moiré fringes while one of them, mounted on a carriage, moves over the other. The moiré pattern then controls the recording of a third grating mounted on the same carriage. This new grating is therefore smoothed by the integration produced by the moiré system over its effective aperture. Two such gratings are then used in the same system to produce another improved generation. In this way, the quality of the gratings can be improved until a limit is set by emulsion creep and considerations of mechanical and thermal stability.

In addition to these linear gratings, the Division demonstrated radial gratings with a moiré system to interpret angular movements. Eventually these gratings will be reproduced photographically, so providing the engineer with a simple accurate method of obtaining digitized information about both radial and linear movement.

The Mathematics Division exhibited DEUCE, its high-speed digital computer. The machine has solved some varied problems during the year, including work on cancer research, the examination of certain vibrational modes of molecules and the stability of aircraft in conditions of flutter.

The Metallurgy Division showed some work on the mechanism of brittle fracture. It has been found that brittle fracture occurs either by separation along grain boundaries or through crystals along cleavage planes. In the former case, two surfaces are formed from one previous interface, and the extra energy, corresponding to the difference between the new surfaces and the original interface, must be supplied for the fracture to occur. This energy difference is often a significant term; hence materials with lowsurface and grain.boundary energies will have a greater tendency to fracture along the boundary than those with high energies. Experiments have been carried out with copper to which increasing amounts of antimony had been added. It was found that the addition of antimony reduced the interfacial energies of the copper-1 $1 \frac{1}{2}$ per cent by weight lowers the energy by more than 80 per cent. It is thought, therefore, that the weakness at the grain boundaries can be associated with the effect of the antimony in lowering the interfacial energies of copper and, therefore, the energy requirements for fracture.

Niobium, with its high melting point, good strength at high temperatures and excellent ductility, is an attractive metal. It suffers, however, from the disadvantage that it easily absorbs gases at high temperatures, particularly hydrogen, with subsequent embrittlement. X-ray diffraction photographs of samples of niobium with known hydrogen contents have been taken. At low temperatures, some hydrogen is absorbed in niobium, and the rest is in the form of a hydride with a structure derived from the cubic lattice of niobium by a simple distortion. On heating, the degree of distortion diminishes and the hydride progressively dissolves in the niobium. At $300^{\circ} \mathrm{C}$. a simple cubic solution of hydrogen in niobium remains. These changes may well have significant effects on the mechanical properties of the metal and on the amount of hydrogen contamination that can be tolerated.

The work of the two Divisions, Applied Physics and Basic Physics, was displayed under the common heading 'Physics'. There have been some advances in bomb calorimetry. The original National Physical

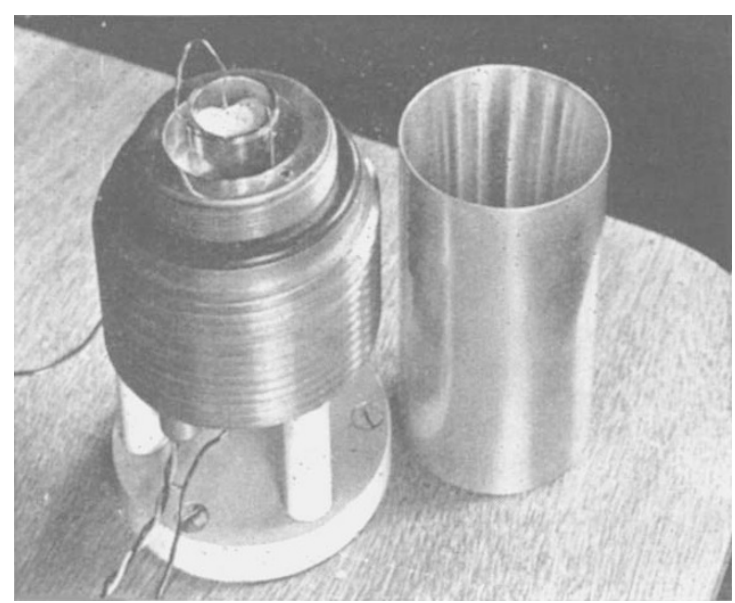

Fig. 2. National Physical Laboratory copper aneroid bomb calorimeter showing the platinum crucible, firing leads and shield
(Crown copyright reserved)

Laboratory bomb calorimeter is unnecessarily precise for the determination of the calorific value of fuels, and the experiments conducted in it take considerable time. Hence, the Laboratory has built a copper aneroid bomb which dispenses with water as a thermal reference (Fig. 2). It needs only four temperature measurements in each determination and its lower thermal capacity enables experiments to be conducted more quickly. The aneroid principle is capable of high accuracy, and a third calorimeter with a silver bomb has been constructed to check the heat of combustion of benzoic acid, the common standard of all such determinations. The original calorimeter was recently used to measure the stored energy in graphite samples taken from the Windscale atomic pile by comparing the heats of combustion of irradiated and unirradiated samples.

An elegant multi-channel pulse-height analyser was displayed. This instrument, when fed with amplified pulses originating, for example, from a proportional counter, will produce a pulse-amplitude spectrum. It was built in collaboration with the Control Mechanisms and Electronics Division concurrently with a similar instrument made by the Admiralty Research Laboratory, Teddington. The analyser operates by converting amplitude to time and then passing the signals of different duration into different channels. It will also provide the difference between two inputs or subtract the background signal measured over the same time interval as the total signal.

Among other exhibits was a demonstration of a possible technique for measuring absolute temperature by determining the Johnson noise generated in a resistor. Such a 'noise thermometer' is theoretically capable of operating over a wide range of temperatures and pressures. Its accuracy is limited by the internal noise of the input valves of the amplifier. It appears that a comparison of noise voltages to 0.05 per cent may be possible, and a resistor has been built with a low enough leakage at $1,100^{\circ} \mathrm{C}$. to exploit this accuracy.

Marine engineers need accurate methods of determining the actual power absorbed by propellers. The Ship Division showed a new torsionmeter which can be attached to propeller shafts of $2-6$ in. diameter without having to unship the shaft. The instrument uses strain gauges arranged in a four-arm bridge 
attached by adhesive to the propeller shaft. The arrangement is such that it responds to torsional strains but not to bending strains. The signals are taken off the rotating shaft through silver-plated slip-rings and silver-graphite brushes. They are amplified and recorded on a null-balance strain meter. The ring assembly incorporates a revolution counter so that the rate of rotation of the shaft can be measured at the same time as the torque. The accuracy of the instrument is \pm 1 per cent for torque determination and \pm 0.2 per cent for speed of rotation.

The Standards Division incorporates the old Metrology Division and parts of the Physics and Electricity Divisions. It had a number of displays showing the value of interferometry, both optical and micro-wave, to science and industry. In 1960, the platinum-iridium bar which is the metric standard will probably be superseded by an 'intangible metre' defined as 1650763.73 times the wave-length in vacuo of the orange radiation of krypton- 86 . This standard will be not only reproducible in any labor. atory, but also it is likely to be about a hundred times as precise as the present line standard. Interferometric methods used in conjunction with this new standard were demonstrated. The Laboratory announces the results of a new determination of the velocity of electromagnetic radiation-in this case not light but $4-\mathrm{mm}$. micro-waves. The velocity in free space in vacuo is $299792.50 \mathrm{~km}$. per sec., with a standard deviation of 3 parts in $10^{7}$. The determination was made with a micro-wave interferometer which measured the wave-length of the radiation. The product of this and the frequency of the source, determined by comparing it with a quartz crystal standard clock, gives the velocity of the radiation. The actual distance over which the velocity was determined was only two metres. The result, with its applications to geodetic surveying, is a contribution to the International Geophysical Year.

To supplement the cæsium atomic standard of frequency, more accurate quartz clocks are required for long-term periods. Investigations have been carried out on the stability of a quartz Essen-ring operating at $4^{\circ} \mathrm{K}$. Preliminary results suggest the damping is much reduced at this temperature and the temperature coefficient between $2^{\circ} \mathrm{K}$. and $4^{\circ} \mathrm{K}$. is less than $1 \times 10^{-8}$. The ring was immersed in liquid helium and the temperature was controlled to $\pm 0.001^{\circ} \mathrm{K}$. by regulating the pressure. The stability of the oscillator may approach 1 part in 10"1. A problem to be solved is the replenishment of liquid helium without mechanical disturbance, and special liquid helium containers designed for this purpose were shown.
A. J. Garratt

\title{
THE SPECIES CONCEPT AMONG VIRUSES
}

\author{
By Dr. C. H. ANDREWES, F.R.S., and DR. P. H. A. SNEATH \\ National Institute for Medical Research, Mill Hill, London, N.W.7
}

T THE term 'species' has been used to describe at least four different ideas:

(I) Linnæus's concept was close to the meaning 'a distinct kind of creature', that is, a group of organisms clearly separate from other groups of organisms, with no intermediates in between, and not itself divisible into distinet sub-groups.

(2) Geneticists consider a species as a 'genetic isolate' (with minor details of difference in definition) since-in organisms reproducing sexually-distinctness usually depends on absence of interbreeding with neighbouring forms.

(3) Palæontologists use the word in sense (1) extended in time, realizing that although obviously the species is continuous in time, with intermediate, ancestral and descendant forms, yet at any one instant the stock is distinct from others, no intermediates being extant.

(4) Systematists commonly use 'species' frankly as 'the most important rank in taxonomy below genus', and this must depend on subjective ideas as to what constitutes a genus, which (like the criteria for all higher ranks) are based mainly on intuitive estimates of degrees of similarity. Methods are, however, now being evolved for expressing this matter of similarity in a quantitative way ${ }^{1,2}$.

There is as yet no general agreement as to which use of the term 'species' is best for general adoption. For viruses, however, only usage (1) can be seriously considered at the moment. Genetic recombination, a form of sexual reproduction, certainly occurs among viruses, but we simply do not know how common it is ; we cannot therefore use the concept of genetic isolate at present in attempting to delimit species of virus. Many viruses are probably mutating and evolving more rapidly than higher organisms; at the same time, our knowledge of their past evolution is negligible; so usage (3) cannot apply. Ideas as to virus taxonomy are at present in an embryonic state, so we can as yet derive little help from usage (4).

\section{Virus Nomenclature}

At the last two International Congresses of Microbiology (Rio de Janeiro, 1950; Rome, 1953), sideration has been given to virus classification and to the usefulness for viruses of a binomial system of nomenclature. As a compromise between two widely divergent views, a plan was put forward in Rome for a 'non-Linnæan' binomial system, provisional and less rigid than the orthodox system. Group names were proposed for some animal viruses and descriptions published: Myxovirus (Andrewes, Bang and Burnet ${ }^{5}$ ), Poliovirus (von Magnus, Gear and Paul ${ }^{6}$ ), Poxvirus (Fenner and Burnet ${ }^{7}$ ). Similar descriptions for a Herpesvirus group and for insect pathogenic viruses are in preparation.

Since their proposal in 1953 and the further pub. lication in 1955, the names Myxovirus and Poliovirus have been widely used, but the 'specific' names have 